\title{
The influence of preventive strategies on the COVID-2019 epidemic in Shenzhen, China
}

\author{
To the Editor:
}

Coronavirus disease 2019 (COVID-19) patients were first reported in Wuhan, in the Hubei province of China, in December 2019; the disease then spread to all provinces of China and $>90$ countries over the next 2 months [1-5]. By 7 March 2020, COVID-19 had infected $>90000$ people and led to $>3000$ deaths [6]. There are 20 million people living in Shenzhen, China, of which the external population from the internal area of China, including Hubei province, account for a large proportion. The high population density, the significant proportion of the external population and the high levels of mobility may have increased the possibility of the COVID-19 outbreak [7, 8]. The first COVID-19 patient in Shenzhen was admitted on 9 January 2020, and 419 cases were confirmed by 7 March 2020, ranking Shenzhen the top city outside Wuhan. Most of these patients were imported cases from the Hubei province, and there was no large-scale transmission or nosocomial infection in Shenzhen, until now.

Therefore, the purpose of this study was to analyse the epidemiology and preventive strategies in Shenzhen in order to understand the main transmission route and effective preventive strategies in cities at risk of imported cases. This may help us to better prevent against the outbreak of potential respiratory infectious diseases like COVID-19 in cities with a heavy population density and a high proportion of external population.

The data in this study were downloaded from the open platform provided by the Shenzhen government (https://opendata.sz.gov.cn/). The number of confirmed cases of COVID-19 began to be released daily on 19 January 2020; by 7 March 2020, 419 cases had been confirmed. Compared with the reports from Wuhan [9], the age of infected population in Shenzhen was younger and decreasing gradually; 33 patients were children. Familial clustering was an important feature of COVID-19 [10]. In Shenzhen, 232 (55.4\%) of the 419 COVID-19 patients came from 86 families, 15 of which had more than three patients, indicating that local transmission in the family environment was an important route of transmission.

Based on the daily new cases, three phases were classified: the slow increase phase from 19 to 28 January 2020; the rapid increase and plateau phase from 29 January to 5 February 2020; and the decline phase, since 6 February 2020 (figure 1). Almost all cases (61 out of 63,96.8\%) in the slow increase phase were patients from Hubei, and two cases were family members of these patients, indicating that the main source of COVID-19 patients were from Hubei province. Compared with the previous period, the majority of patients (174 out of $228,76.3 \%$ ) in the rapid increase and plateau phase were still from Hubei, but infection related to contact with Hubei patients (27 out of $228,11.8 \%$ ), non-Hubei patients (12 out of 228, $5.3 \%$ ) and no contact history ( 14 out of $228,6.1 \%$ ) increased. In the decline phase, the number of daily new confirmed cases decreased significantly, but infection related to contact with Hubei patients (27 out of $128,21.1 \%$ ), non-Hubei patients (18 out of $128,14.1 \%$ ) and no contact history (nine out of $128,7 \%$ ) had an increase tendency.

The preventive strategies can be divided into early prevention and strengthened prevention. Early preventative strategies and measures against COVID-19 included public announcement of the outbreak in Wuhan and preparation for the diagnosis and management of COVID-19 in hospitals.

@ERSpublications

Early identification of imported cases, prevention of family clustering transmission, preventive measures in public areas and strict infection control procedures in hospitals were crucial for successful prevention of COVID-19 in Shenzhen, China https://bit.ly/2UDb5JA

Cite this article as: Yang $\mathrm{K}$, Wang $\mathrm{L}, \mathrm{Li} \mathrm{F}$, et al. The influence of preventive strategies on the COVID2019 epidemic in Shenzhen, China. Eur Respir J 2020; 55: 2000599 [https://doi.org/10.1183/ 13993003.00599-2020]. 

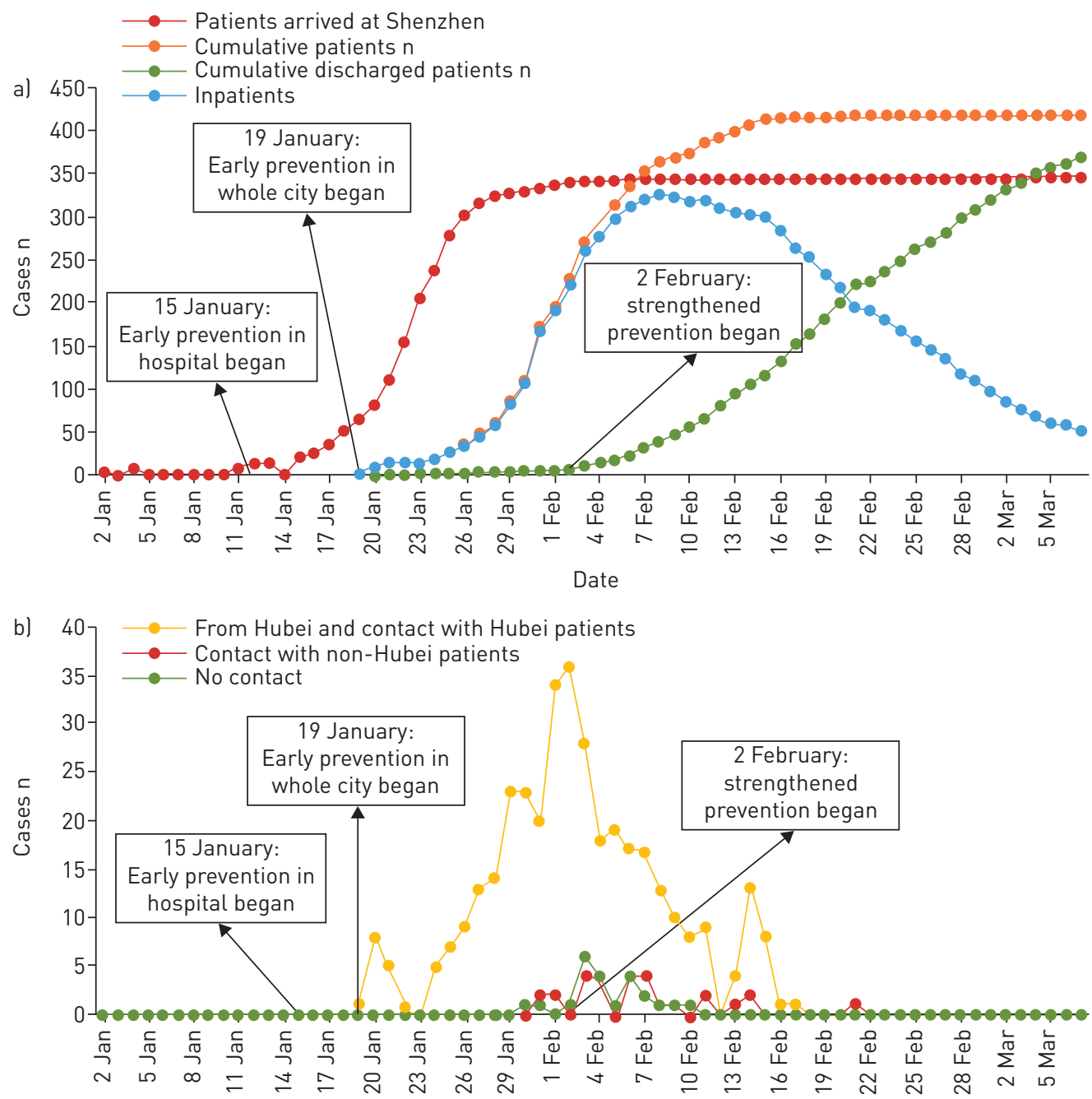

Date

FIGURE 1 Epidemic curve of coronavirus disease 2019 (COVID-19) and prevention strategies in Shenzhen, China, up to 7 March 2020. a) Cumulative number of patients for different indexes. b) Epidemic curve of COVID-19 patients with different infectious patterns.

The hospitals began to prepare for the possible outbreak of COVID-19 on 1515 January 2020. These preparations included training medical staff and epidemiological investigators, hospital disinfection, medical material reserve, outpatient adjustment and medical waste management. All 49 hospitals in Shenzhen set up 24-h fever clinics to screen for possible COVID-19 with nasal or throat swab PCR detection, chest computed tomography (CT) and blood lymphocyte counts. Suspected patients were isolated and observed in single room for each case, with special preparation of infection control measures in the hospital. Confirmed patients were transferred to Shenzhen infectious disease hospitals (designated hospitals for inpatient management of infectious disease) for treatment using a negative-pressure ambulance.

All medical staff involved in the management of COVID-19 patients implemented infection control procedures. For the general population, people entering the hospital were asked to wear masks and take their body temperature. The hospital environment was disinfected regularly; hand-washing equipment and disinfectants were provided in multiple places; indoor air ventilation was strengthened.

As early as 19 January 2020, when the National Health Commission announced the first imported case in Shenzhen, the city began to take the temperature of people at the main city entrances to screen imported cases; this method was also widely used in the prevention of other respiratory infectious diseases, such as influenza in the USA and severe acute respiratory syndrome in China [11,12]. After the release of the first (highest) level response to major public health emergencies in Guangdong on 23 January 2020, all Chinese 
New Year entertainment was cancelled and many public places were temporarily closed in Shenzhen, such as the market, libraries and gymnasiums. Other necessary public places were disinfected regularly, including the airport, the train station, the port, the freeway entrance and the resident community entrance. The employees in these places had health examinations. People who came to these places had to wear masks and take their body temperature. These measures were important in blocking the spread of COVID-19.

Millions of people returned to Shenzhen from all over the country after Chinese New Year, which may have led to the imported cases and potential transmission. Therefore, strengthened measures were implemented on 2 February 2020. These measures included: isolating all new arrivals from epidemic areas for medical observation for 14 days; informing all people in communities when there were confirmed cases; and using big data and information technology to track the places visited in the last 14 days and identify the contacted persons of the confirmed cases.

The preventive strategies and measures implemented in Shenzhen were supposed to block the transmission of COVID-2019. The newly diagnosed COVID-19 cases reached their peak around 31 January 2020, which was 7 days after the peak date of cases arriving at Shenzhen (mainly patients in the incubation period from Hubei) and around 10 days after implementation of the serial early preventive strategy. Taking the incubation period (3-7 days, with mean of 3.7 days) into account and the time between symptom onset and confirmation of diagnosis (6 days on average) [13, 14], the peak of new confirmed cases coincided with the implementation of serial preventative strategies, indicating that these measures were effective in preventing transmission of COVID-19 in Shenzhen. The lockdown of Wuhan and other cities in Hubei on 23 January 2020 and the next few days was also important for the reduction of imported cases in Shenzhen, which reduced the imported cases and the difficulty of prevention in Shenzhen. Most of the imported cases after the lockdown left Hubei before January 23 and arrived at Shenzhen later, which led to increasing imported cases in the few days after January 23 (figure 1). There was no infection among medical staff or patients infected in the hospital environment in Shenzhen until 7 March 2020.

In conclusion, with the major outbreak of COVID-19 in nearby province of Hubei, Shenzhen, which is a city that has a high population density, a large proportion of external population and significant mobility, had consider imported cases and the risk of spreading the disease throughout the city. The implementation of early preventative strategies in Shenzhen was successful, allowing early identification of COVID-19 cases and preventing a major outbreak in Shenzhen. Early identification of imported cases, prevention of transmission through family clustering, preventative measures in public areas and very strict infection control procedures in the hospital setting were crucial to the successful control of outbreak in Shenzhen.

Kai Yang ${ }^{1,2,3}$, Lingwei Wang ${ }^{1,2,3}$, Furong $\mathrm{Li}^{1,2}$, Dandan $\mathrm{Chen}^{1,2}, \mathrm{Xi} \mathrm{Li}^{1,2}$, Chen Qiu ${ }^{1,2,4}$ and Rongchang Chen ${ }^{1,2,4}$ ${ }^{1}$ Dept of Respiratory and Critical Care Medicine, Shenzhen Key Laboratory of Respiratory Diseases, Shenzhen Institute of Respiratory Diseases, Second Clinical Medical College of Jinan University (Shenzhen people's Hospital), Shenzhen, China. ${ }^{2}$ Dept of Respiratory and Critical Care Medicine, First Affiliated Hospital of South University of Science and Technology, Shenzhen, China. ${ }^{3}$ Equal contribution. ${ }^{4}$ Equal contribution as senior author.

Correspondence: Rongchang Chen, Dept of Respiratory and Critical Care Medicine, Second Clinical Medical College of Jinan University (Shenzhen people's Hospital), 1017 Dongmen North Rd, Shenzhen 518000, China.

E-mail: chenrc@vip.163.com

Received: 9 March 2020 | Accepted after revision: 18 March 2020

Conflict of interest: None declared.

Support statement: This work was supported by the emergency research project of Zhejiang University (Hangzhou, China) for COVID-19 infection (number 2020XGZX024). Funding information for this article has been deposited with the Crossref Funder Registry.

\section{References}

1 Chang D, Lin M, Wei L, et al. Epidemiologic and clinical characteristics of novel coronavirus infections involving 13 patients outside Wuhan, China. JAMA 2020; in press [https://doi.org/10.1001/jama.2020.1623].

2 Holshue ML, DeBolt C, Lindquist S, et al. First case of 2019 novel coronavirus in the United States. $N$ Engl J Med 2020; 382: 929-936.

3 Li Q, Guan X, Wu P, et al. Early transmission dynamics in Wuhan, China, of novel coronavirus-infected pneumonia. N Engl J Med 2020; 382: 1199-1207.

4 Wang C, Horby PW, Hayden FG, et al. A novel coronavirus outbreak of global health concern. Lancet 2020; 395: $470-473$.

5 Zhu N, Zhang D, Wang W, et al. A novel coronavirus from patients with pneumonia in China, 2019. N Engl J Med 2020; 382: 727-733. 
6 Team TNCPERE. The epidemiological characteristics of an outbreak of 2019 novel coronavirus diseases (COVID-19) in China. Zhonghua Liu Xing Bing Xue Za Zhi 2020; 41: 145-151.

7 Wang Y, Wang X, Liu X, et al. Epidemiology of imported infectious diseases, China, 2005-2016. Emerging Infect Dis 2018; 25: 33-41.

8 Lewnard JA, Reingold AL. Emerging challenges and opportunities in infectious disease epidemiology. Am J Epidemiol 2019; 188: 873-882.

9 Huang C, Wang Y, Li X, et al. Clinical features of patients infected with 2019 novel coronavirus in Wuhan, China. Lancet 2020; 395: 497-506.

10 Chan JF, Yuan S, Kok KH, et al. A familial cluster of pneumonia associated with the 2019 novel coronavirus indicating person-to-person transmission: a study of a family cluster. Lancet 2020; 395: 514-523.

11 Priest PC, Jennings LC, Duncan AR, et al. Effectiveness of border screening for detecting influenza in arriving airline travelers. Am J Public Health 2013; 103: 1412-1418.

12 Pitman RJ, Cooper BS, Trotter CL, et al. Entry screening for severe acute respiratory syndrome (SARS) or influenza: policy evaluation. BMJ 2005; 331: 1242-1243.

13 Wang $\mathrm{D}, \mathrm{Hu} \mathrm{B}$, $\mathrm{Hu} \mathrm{C}$, et al. Clinical characteristics of 138 hospitalized patients with 2019 novel coronavirus-infected pneumonia in Wuhan, China. JAMA 2020; in press [https://doi.org/10.1001/jama.2020.1585].

14 Guan WJ, Ni ZY, Hu Y, et al. Clinical characteristics of coronavirus disease 2019 in China. N Engl J Med 2020; in press [https://doi.org/10.1056/NEJMoa2002032]. 